\title{
Failing heart or failing liver? Reverse cavo-portal shunt with bleeding gastric varices, in severe congestive heart failure. A novel report in literature
}

\begin{abstract}
Upper gastrointestinal (GI) bleedings of esophageal or gastric varices occur in the settings of portal hypertension due to well-known porto-systemic shunts. In this communication we report a previously unrecognized pathophysiological mechanism of gastric varices formation, via spontaneous reno-gastric shunt, as a result of Inferior Vena Cava (IVC) hypertension, rather than portal hypertension. We report a case of 54years old male with severe chronic congestive heart failure and bleeding gastric varices. Cardiac cirrhosis was initially suspected as a cause for his condition. However, direct venography measurements showed high central venous and portal pressures without significant porto-systemic gradient, and hepatopetal flow direction from the IVC though left renal vein and via spontaneous reno-gastric shunt towards gastric varices. This paradoxical cavo-portal, collateralization pathway should be considered in differential diagnosis of upper GI bleeding in patients with severe congestive heart failure, it can be diagnosed by venography and treated by trans-caval embolization.
\end{abstract}

Volume I Issue I - 2015

\author{
David Aranovich,' Oleg Kaminsky,' \\ Alexander Belenky ${ }^{2}$ \\ 'Department of Surgery, Rabin Medical Center, Israel \\ ${ }^{2}$ Department of Radiology, Rabin Medical Center, Israel
}

Correspondence: David Aranovich, Department of Surgery, Rabin Medical Center, Beilinson Hospital, 39 Jabotinsky Street, 49100 Petah Tikva, Israel, Tel +972(0)58-4567-650, Fax +972(0)3-9376262, Email aradair@yahoo.com

Received: June 05, 2015 | Published: July 16, 2015

\section{Introduction}

Variceal upper gastrointestinal (GI) bleeding is a well-recognized complication of liver cirrhosis. Several other less common condition of non-cirrhotic portal hypertension have been implicated in formation of esophageal and/or gastric varices, such as congenital malformations, portal and splenic vein thrombosis, various systemic and infectious causes. ${ }^{1}$ In this report we present a case of variceal gastrointestinal bleeding, secondary to severe heart failure. As a result of severe Superior Vena Cava (SVC) congestion, the blood flow was partially diverted through left renal vein and spontaneous reno-gastric shunt into portal system, thus creating gastric varices of systemic, rather than portal origin.

\section{Case presentation}

A fifty four years old male presented with intermittent upper gastrointestinal (GI) bleeding. His past medical history was significant for severe heart failure, coronary artery bypass surgery, mechanical aortic and mitral valve replacement, chronic atrial fibrillation, end stage renal failure on hemodialysis, and pacemaker implantation. He had no past history of liver disease, and his liver viral serology was negative. The patient was passing dark stool, and required daily blood transfusions and withdrawal of anticoagulation upon initial stabilization. Upper gastrointestinal endoscopy showed varices at the cardiac portion of the stomach with stigmata of recent bleeding. Due to anatomic location of the varices in the cardia, endoscopic band ligation was not technically possible. Abdominal CT scan showed varices in the vicinity of gastric fundus with spontaneous collateral venous shunt connecting left renal vein and stomach (Figures 1-3).

Giving the patient's clinical history of severe heart failure and upper GI bleeding due to gastric varices, we presumed that the patient developed portal hypertension, secondary to cardiac cirrhosis. Since, endoscopic treatment of the varices was not feasible, the patient was planned for Transjugular Intrahepatic Portosystemic Shunt (TIPS) under general anesthesia. At the beginning of the procedure, the central venous and hepatic wedge pressures were accessed via right transjugular route. The pressure measurements were as following: central venous pressure was 25 and $27 \mathrm{~mm} \mathrm{Hg}$ in right atrium and in inferior vena cava respectively. Hepatic vein wedge pressure, representing portal vein pressure, was $30 \mathrm{~mm} \mathrm{Hg}$, with measured Portosystemic Venous Gradient (PSVG) of $5 \mathrm{~mm} \mathrm{Hg}$. Due to extremely high central and right heart pressures, and absence of significant PSVG, the TIPS procedure was aborted. Then, we attempted direct embolization of the varices via spontaneous gastro-renal shunt. The right femoral vein was accessed and venography performed. To our surprise, we observed upward (hepatopetal) blood flow through the spontaneous shunt towards the gastric varices (Figure 4) (Figure 5). The varices were successfully embolized via the shunt with resolution of patient's bleeding. After successful embolization of gastric varices the patient stopped bleeding, and was eventually discharged home. Unfortunately he died three weeks later because of worsening end heart failure.

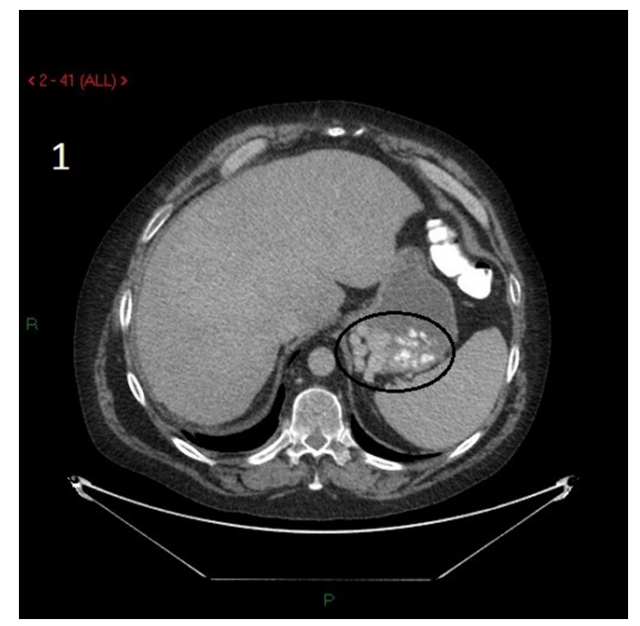

Figure I Abdominal CT scan, transverse view, showing a conglomerate of varices outside and inside the stomach wall (outlined with a circle). 


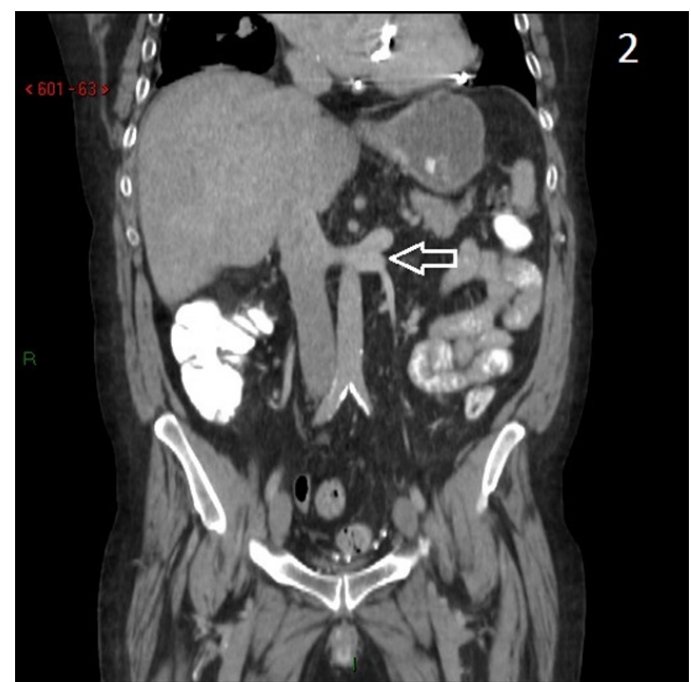

Figure 2 CT scan, coronary view. There is a spontaneous venous shunt (arrow), taking off the left renal vein.

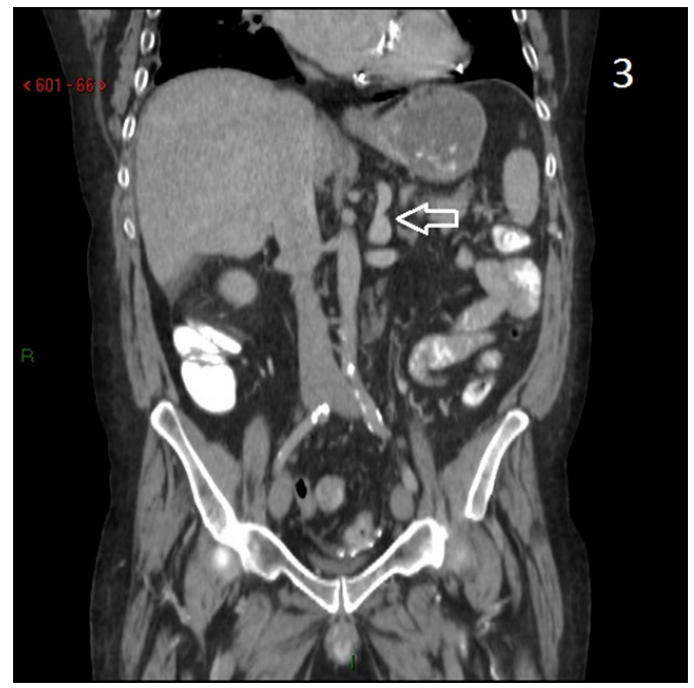

Figure 3 Additional coronary CT view, showing the direction of the shunt towards the stomach.

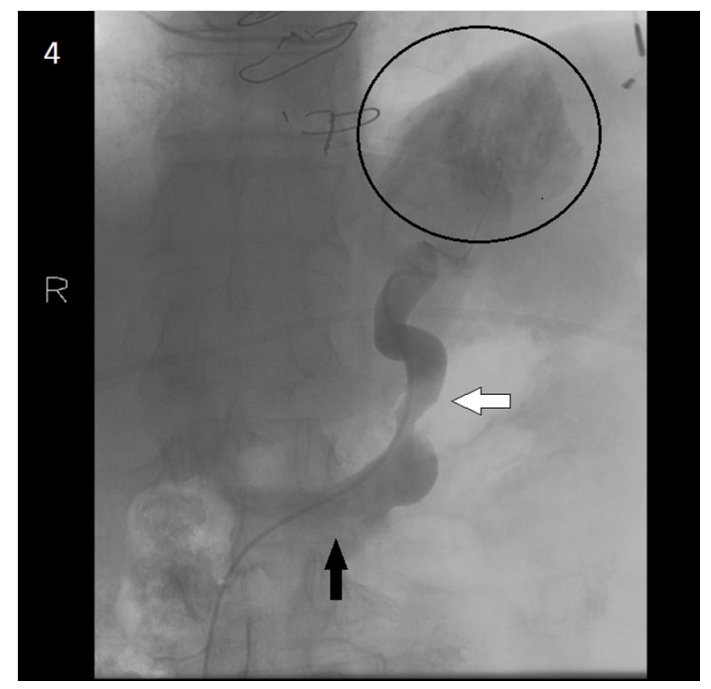

Figure 4 Venography. The catheter enters Inferior Vena Cava and passes to the shunt (transparent arrow) via the Left RenalVein (solid arrow). The varices are outlined (circle).

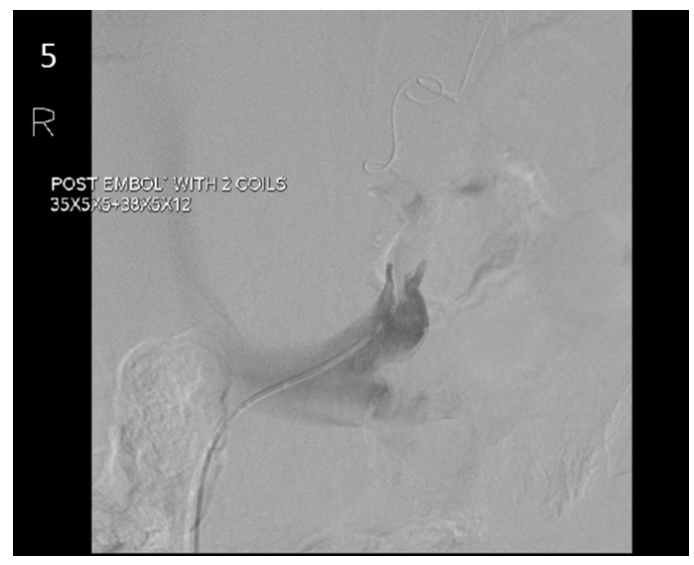

Figure 5 Venography.After transcatheter embolization of the shunt with coils.

\section{Discussion}

Esophageal or gastric varices develop as a result of resistance to portal blood flow and may lead to complications such as variceal bleeding. The most common etiology of increased resistance to portal blood flow is intrinsic hepatic causes, such as cirrhosis. Other extra- or intra-hepatic conditions may result in non-cirrhotic portal hypertension syndromes. ${ }^{1}$ These conditions include prehepatic causes, such as, portal or splenic vein thrombosis, vascular malformations, and splenomegaly in lymphoma or Gaucher's disease. Post-hepatic causes include obstruction of the inferior vena cava in Budd-Chiari syndrome, constrictive pericarditis and restrictive cardiomyopathy. All these conditions may result in formation of gastro-esophageal varices due to increased pressure in the portal system. Rarely esophageal varices may occur in the absence of portal hypertension. "Downhill" varices as a consequence of Superior Vena Cava (SVC) obstruction have been described is small case series, mainly in chronic hemodialysis patients. "Downhill" varices develop when an obstruction in the superior vena cava flow forces the blood towards the heart, using collateral mediastinal pathways to the inferior vena cava. ${ }^{3,4}$ All known pathways of venous outflow collateralization of the upper gastrointestinal tract in case of varices, universally involve either porto-systemic shunts (portal hypertension), or rarely SVC obstruction ("downhill" varices). In these cases the blood finds its way Towards the heart by means of collateral pathways.

We present the case of variceal gastrointestinal bleeding in a patient with severe congestive heart failure, in which the blood ran Away from heart to the portal venous system. Giving the patient's history of long standing severe congestive heart failure, the gastric varices were initially attributed to presumed decompensated cardiac cirrhosis. Interventional radiology plays an important role in achieving hemostasis in patient with acute variceal bleeding. This patient failed conventional pharmacological and endoscopic therapy, thus TIPS was opted as salvage method to control the ongoing bleeding and prevent recurrences. TIPS functions as low resistance channel between portal vein and Inferior Vena Cava (IVC), thus effectively reducing portosystemic venous gradient (PSVG). Invasive right atrium and central pressures measurements were extremely high, displaying severe right heart failure, and no portal hypertension was demonstrated by direct measurement. Porto-systemic venous gradient of $5 \mathrm{~mm} \mathrm{Hg}$, essentially excluded portal hypertension as a possible cause of variceal formation. Subsequent venography showed "away from the heart" collateralization of venous blood flow via spontaneous shunt between left renal vein and gastric venous tributaries, thus forming varices at the gastric cardia. The presented case shows that under certain 
conditions extremely elevated cardiac preload can force blood into gastric venous system via natural occurring shunts, thus producing variceal gastrointestinal bleeding. This route of collateralization bypasses high pressure central system into low pressure portal circulation. To the best of our knowledge, this type of reno-gastric, cavo-portal shunt with bleeding gastric varices, as a complication of right sided heart failure, has never been reported in English literature.

\section{Acknowledgements}

None.

\section{Conflict of interest}

Author declares that there is no conflict of interest.

\section{References}

1. Schouten JN, Garcia-Pagan JC, Valla DC, et al. Idiopathic noncirrhotic portal hypertension. Hepatology. 2011;54(3):1071-1081.

2. Hussein FA, Mawla N, Befeler AS, et al. Formation of downhill esophageal varices as a rare but serious complication of hemodialysis access: a case report and comprehensive literature review. Clin Exp Nephrol. 2008;12(5):407-415.

3. Pop A, Cutler AF. Bleeding downhill esophageal varices: a complication of upper extremity hemodialysis access. Gastrointest Endosc. 1998;47(3):299-303.

4. Areia M, Romaozinho JM, Ferreira M, et al. Downhill varices. A rare cause of esophageal hemorrhage. Rev Esp Enferm Dig. 2006;98(5):359-361. 